\title{
Analysis and Adaptive Estimation of the Registration Noise Distribution in Multitemporal VHR Images
}

\author{
Francesca Bovolo, Member, IEEE, Lorenzo Bruzzone, Senior Member, IEEE, and \\ Silvia Marchesi, Student Member, IEEE
}

\begin{abstract}
This paper analyzes the problem of change detection in very high resolution (VHR) multitemporal images by studying the effects of residual misregistration [registration noise $(\mathrm{RN})]$ between images acquired on the same geographical area at different times. In particular, according to an experimental analysis driven from a theoretical study, the main effects of $\mathrm{RN}$ on VHR images are identified and some important properties are derived and described in a polar framework for change vector analysis. In addition, a technique for an adaptive and unsupervised explicit estimation of the $R N$ distribution in the polar domain is proposed. This technique derives the RN distribution according to both a multiscale analysis of the distribution of spectral change vectors and the Parzen windows method. Experimental results obtained on simulated and real multitemporal data sets confirm the validity of the proposed analysis, the reliability of the derived properties on $\mathrm{RN}$, and the effectiveness of the proposed estimation technique. This technique represents a very promising tool for the definition of changedetection methods for VHR multitemporal images robust to RN.
\end{abstract}

Index Terms - Change detection, change vector analysis (CVA), multitemporal image analysis, registration noise ( $R N)$, remote sensing, very high resolution (VHR) images.

\section{INTRODUCTION}

$\mathbf{T}$ HE ever-increasing availability of remote-sensing images regularly acquired by satellites over the same geographical area makes the analysis of multitemporal data (and the related applications) one of the most interesting research topics for the remote-sensing community. Multitemporal images represent a valuable information source for performing the detection of changes occurred on the Earth surface at different scales. Change-detection techniques generally compare two images acquired at different times by assuming that they are similar to each other except for the presence of changes occurred on the ground. Unfortunately, this assumption is seldom completely satisfied due to differences in atmospheric and sunlight conditions, as well as in the sensor acquisition geometry. In order to satisfy the similarity assumption, preprocessing steps are required, including: image coregistration, radiometric and geometric corrections, and noise reduction. Among the others,

Manuscript received July 31, 2008; revised January 8, 2009. First published June 5, 2009; current version published July 23, 2009.

The authors are with the Department of Information Engineering and Computer Science, University of Trento, 38050 Trento, Italy (e-mail: lorenzo. bruzzone@ing.unitn.it).

Color versions of one or more of the figures in this paper are available online at http://ieeexplore.ieee.org.

Digital Object Identifier 10.1109/TGRS.2009.2017014 coregistration plays a fundamental role as it allows one to obtain a pair of images where corresponding pixels are associated with the same position on the ground. However, in practice, it is not possible to obtain a perfect alignment between images acquired at different times. This may significantly affect the accuracy of the change-detection process. The coregistration procedure becomes more complex and critical (and therefore intrinsically less accurate) when very high resolution (VHR) images acquired by the last generation sensors (e.g., WorldView, Ikonos, Eros, Quickbird, SPOT-5) are considered. These images can be acquired with different view angles and often show different geometrical distortions that, even after proper geometric corrections, strongly affect the precision of the registration process, thus resulting in a significant residual registration noise (RN). This noise sharply decreases the accuracy of the changedetection process.

In this paper, large attention has been devoted to the development of advanced registration techniques, particularly for what concerns medium-resolution multitemporal and multisensor images [1]-[5]. Moreover, some studies exist on the effects of misregistration on the change-detection accuracy [6]-[11] and on the development of change-detection techniques less sensitive to problems due to misregistration [12], [13]. Nonetheless, in our knowledge, few attentions have been devoted to study the effects and the properties of RN in VHR images.

This paper aims at analyzing the properties of $\mathrm{RN}$ in multitemporal VHR images in order to formulate an adaptive technique for the explicit estimation of the distribution of residual RN between multitemporal images. This distribution is a starting point for the development of novel change-detection techniques robust to such source of noise. This paper is developed within a polar framework for change vector analysis (CVA) recently introduced in the literature for change detection in medium resolution multispectral images [14]. The definition of this framework is based on the analysis of the distribution of spectral change vectors ( $\mathrm{SCVs}$ ) computed according to the CVA technique in the polar domain. In this context, the novel contributions of this paper consist in: 1) the analysis of the effects of RN in multitemporal and multispectral VHR images according to the study of the statistical distribution of SCVs; 2) the definition of the properties of RN in VHR images; and 3) the formulation of an adaptive and distribution-free technique for the estimation of the distribution of the $\mathrm{RN}$ in the polar domain. This last technique exploits the Parzen windows estimation 
procedure and takes advantage from both a multiscale decomposition of multitemporal images and the properties derived in the first part of this paper. The experiments carried out on simulated and real multitemporal images confirm the validity of the theoretical analysis and the effectiveness of the proposed technique, which represents a valuable tool for the development of reliable change-detection techniques for multitemporal and multispectral VHR images. It is worth noting that the proposed method can be suitable also for the analysis of optical data at lower resolution; however, we consider only very high geometrical resolution images as the impact of misregistration on this kind of data is more relevant.

This paper is organized into six sections. The next section briefly recalls the notation and the background of the polar framework proposed in [14]. Section III describes the experimental setup for the study of the properties of RN on simulated multitemporal VHR images. Section IV derives and defines the properties of RN. Section V illustrates the proposed approach to the estimation of the distribution of $\mathrm{RN}$ in the polar domain. Section VI presents the validation on real multitemporal Quickbird images of both the derived properties and the proposed technique for the estimation of the RN. Finally, Section VII draws the conclusions of this paper.

\section{NOTATION AND BACKGROUND}

In order to analyze the effects of the $\mathrm{RN}$, we take advantage from the theoretical polar framework defined for unsupervised change detection based on CVA proposed in [14]. In particular, we derive the properties and adaptively estimate the distribution of RN according to the behavior of SCVs in such polar domain. ${ }^{1}$ In the following, we briefly recall the main concepts of this framework.

Let us consider two VHR multispectral images $\mathbf{X}_{1}$ and $\mathbf{X}_{2}$ (e.g., WorldView, Ikonos, Eros, Quickbird, SPOT-5 images) acquired over the same geographical area at different times $t_{1}$ and $t_{2}$, respectively. Let us assume that these images do not show significant radiometric differences. Let $\Omega=\left\{\omega_{\mathrm{n}}, \Omega_{\mathrm{c}}\right\}$ be the set of classes of changed and unchanged pixels to be identified. In greater detail, $\omega_{\mathrm{n}}$ represents the class of unchanged pixels and $\Omega_{\mathrm{c}}=\left\{\omega_{\mathrm{c}_{1}}, \ldots, \omega_{\mathrm{c}_{K}}\right\}$ the set of the $K$ possible classes (kinds) of changes occurred in the considered area. For simplicity, the whole analysis on the $\mathrm{RN}$ properties is carried out considering a 2-D feature space (however, it can be generalized to the case of more features, see [14] for details). In this manner, it is possible to represent the information in a 2-D domain and to better understand the implications of the analysis. Let $\mathbf{X}_{\mathrm{D}}$ be the multispectral difference image computed according to the CVA technique by subtracting the spectral feature vectors associated with each corresponding spatial position in the two considered images. $\mathbf{X}_{\mathrm{D}}$ is a multidimensional image made up of SCVs defined as

$$
\mathbf{X}_{\mathrm{D}}=\mathbf{X}_{2}-\mathbf{X}_{1}
$$

\footnotetext{
${ }^{1}$ It is worth noting that in this paper, we do not present a change-detection technique, but we focus the analysis on the RN properties and on the definition of a method for an adaptive estimation of it.
}

Under the assumption of 2-D feature vectors, the change information contained in the SCVs can be univocally described by the change vector magnitude $\rho$ and direction $\vartheta$ defined as

$$
\vartheta=\tan ^{-1}\left(\frac{X_{1, \mathrm{D}}}{X_{2, \mathrm{D}}}\right) \quad \rho=\sqrt{\left(X_{1, \mathrm{D}}\right)^{2}+\left(X_{2, \mathrm{D}}\right)^{2}}
$$

where $X_{b, \mathrm{D}}$ is the random variable representing the $b$ th component (spectral channel) of $\mathbf{X}_{\mathrm{D}}(b=\{1,2\})$. Finally, let us define the magnitude-direction domain $M D$ (in which all the SCVs of a given scene are included) as

$$
M D=\left\{\rho \in\left[0, \rho_{\max }\right] \text { and } \vartheta \in[0,2 \pi]\right\}
$$

where $\rho_{\max }$ is the highest magnitude of SCVs in the considered images.

From the theoretical analysis reported in [14] and under the aforementioned assumptions, it is expected that in the polar representation unchanged and changed SCVs result in separated clusters. Unchanged SCVs show a low magnitude and are uniformly distributed with respect to the direction variable. In the polar domain the region associated with them is the circle of no-changed pixels $C_{\mathrm{n}}$, defined as

$$
C_{\mathrm{n}}=\{\rho, \vartheta: 0<\rho \leq T \text { and } 0 \leq \vartheta<2 \pi\} .
$$

This circle is centered at the origin and has a radius equal to the optimal (in the sense of the theoretical Bayesian decision theory) threshold $T$ that separates unchanged from changed pixels. On the opposite, changed SCVs are expected to show a high magnitude. The region associated with them in the polar domain is the annulus of changed pixels $A_{\mathrm{c}}$, which is defined as

$$
A_{\mathrm{c}}=\left\{\rho, \vartheta: T \leq \rho<\rho_{\max } \text { and } 0 \leq \vartheta<2 \pi\right\} .
$$

This annulus has inner radius $T$ and outer radius given by the maximum among all possible magnitudes for the considered pair of images $\left(\rho_{\max }\right)$. As unchanged SCVs show preferred directions according to the kind of change occurred on the ground, different kinds of change can be isolated with a pair of threshold values $\left(\vartheta_{k_{1}}\right.$ and $\left.\vartheta_{k_{2}}\right)$ in the direction domain. Each pair of thresholds identifies, in the annulus of changed pixels $A_{\mathrm{c}}$, an annular sector $S_{k}$ of change $\omega_{k} \in \Omega_{\mathrm{c}}$, defined as

$S_{k}=\left\{\rho, \vartheta: \rho \geq T\right.$ and $\left.\vartheta_{k_{1}} \leq \vartheta \leq \vartheta_{k_{2}}, 0 \leq \vartheta_{k_{1}}<\vartheta_{k_{2}} \leq 2 \pi\right\}$.

All the mentioned regions are shown in Fig. 1. The reader is referred to [14] for further details on both the polar framework and the general properties of SCVs in this kind of representation.

\section{Design of the ANALysis AND EXPERIMENTAL SETUP}

The objective of this paper is to study the effects of misregistration within the framework presented in the previous section in order to derive its properties and to define a procedure for an adaptive estimation of the distribution of RN. As previously mentioned, residual misregistration affects multitemporal data and represents an important source of noise. In particular, this noise becomes more relevant when dealing with VHR 


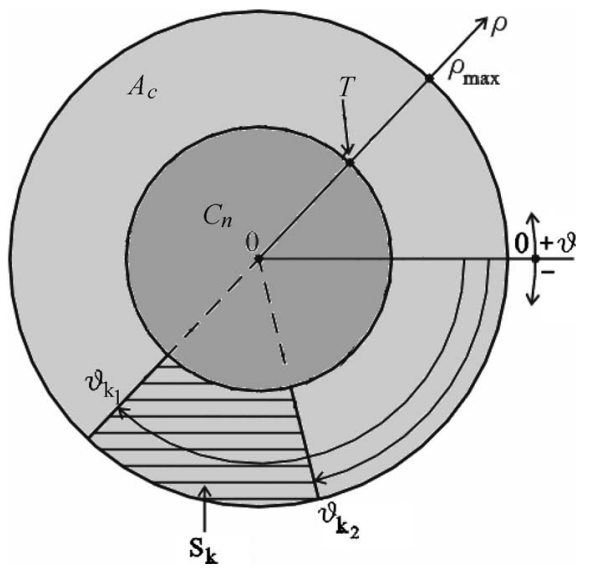

Fig. 1. Representation of the regions of interest in the CVA polar framework.

images, as the process of coregistration is more complex and critical. Indeed, images acquired by VHR sensors of the last generation can be acquired with different view angles and often show different geometrical distortions that strongly affect the registration process. Thus, they result in a significant amount of residual RN. For this reason, it is very important to study the properties of RN and to define techniques for estimating its distribution (which is a valuable information to be given as input to change-detection methods).

$\mathrm{RN}$ is due to the comparison of pixels that do not represent the same area on the ground in images acquired over the same geographical area at different times. In particular, the most critical component of $\mathrm{RN}$ is related to the pixels that at the two dates belong to different objects/classes on the ground (as discussed in the next section) due to the misalignment between the two images. In fact, these pixels show a behavior similar to the one of real changes, causing misclassification effects in the change-detection process. It follows that it is important to identify these pixels and separate them from pixels associated with true changes in the multitemporal data analysis.

The residual $\mathrm{RN}$ can be modeled as the effect of different types of transformations between the images, such as scale variation, rotation, translation, and skew [6]. In this section, for space constraints, only examples modeling the $\mathrm{RN}$ as a translational effect are reported; however, this choice is reasonable as, according to [6], nontranslational effects show (from a statistical viewpoint) a behavior similar to that of the translational ones. This behavior is confirmed by experimental results obtained with misregistered data sets generated considering relative rotation and rototranslation, which are not reported here for space constraints.

In order to study the $\mathrm{RN}$ in the polar CVA domain, several data sets have been selected by considering: 1) very high geometrical resolution images acquired by different sensors (i.e., Quickbird, Ikonos, and Pleiades simulator) and 2) areas with different characteristics, representative of the most frequent land-cover types (i.e., urban, rural, and forestry). Three different experiments have been defined to understand the behavior of $\mathrm{RN}$ on unchanged and changed pixels when the misalignment between images increases and the resolution level decreases. To avoid intrinsic differences between images typical of real multitemporal data sets (e.g., atmospheric differences, etc.), in the first phase of the analysis, a single-date image has been considered for each data set, while the second acquisition has been simulated. The analysis carried out on the single-date data sets is then extended to real multitemporal images in Section VI.

In the following, we describe the experiments considering the analysis conducted on a Quickbird image acquired on the city of Trento (Italy) in July $2006\left(\mathbf{X}_{1}\right)$. The selected test site is a section of a full scene including both rural and urban areas [Fig. 2(a)]. Results obtained on other data sets (which contain areas with other characteristics and images acquired by other sensors) are very similar to those reported here and, thus, omitted for space constraints. In the following, after an accurate preliminary analysis, among the four available spectral channels, only the red and the near-infrared ones were considered for analyzing the distributions in the polar domain, as they demonstrated to be the most effective in emphasizing the properties of RN (with respect to both changed and unchanged pixels) on the adopted data set. Different choices led to poorer visual representations but to similar conclusions.

\section{A. Experiment 1: Effects of Increasing Misregistration on Unchanged Pixels}

From the considered image $\mathbf{X}_{1}$, different simulated images $\mathbf{X}_{2}$ have been generated introducing some pixels of misregistration according to translations in several directions. This resulted in different multitemporal data sets made up of the original image $\mathbf{X}_{1}$ and of its shifted versions $\mathbf{X}_{2}$. In particular, we considered misregistration between 1 and 6 pixels, which are reasonable values when taking into account large VHR images acquired with different view angles and/or in complex areas. After the application of the CVA, the SCV distributions were analyzed in the polar scatterograms in order to derive the properties of $\mathrm{RN}$ on unchanged pixels. It is worth noting that the application of the CVA technique to $\mathbf{X}_{1}$ and a copy of itself when images are perfectly coregistered leads to a multispectral difference image made up of SCVs with all zero components. Thus, the representation in polar coordinates of SCVs collapses in a single point at the origin. This is no longer valid if the CVA is applied to misregistered images; in this case, the distribution of SCVs in the polar domain corresponds to the distribution of RN (as no changes are present in the considered data set). Fig. 3 shows an example of the behaviors of scatterograms obtained by applying the CVA technique to $\mathbf{X}_{1}$ and its 2- and 6-pixel-shifted versions, respectively. An analysis of these scatterograms allows us to derive the properties of $\mathrm{RN}$ when no changes are present between the considered images (see Section IV).

\section{B. Experiment 2: Effects of Increasing Misregistration on Changed Pixels}

From the considered image $\mathbf{X}_{1}$, a new image $\mathbf{X}_{2}$ has been generated by adding simulated changes. These changes have been accurately introduced in order to be as similar as possible to real changes. In particular, some buildings have been added to the scene [see regions marked with white circles in Fig. 2(b)] taking their geometrical structures and spectral signatures from other real buildings present in the image. All the mentioned 


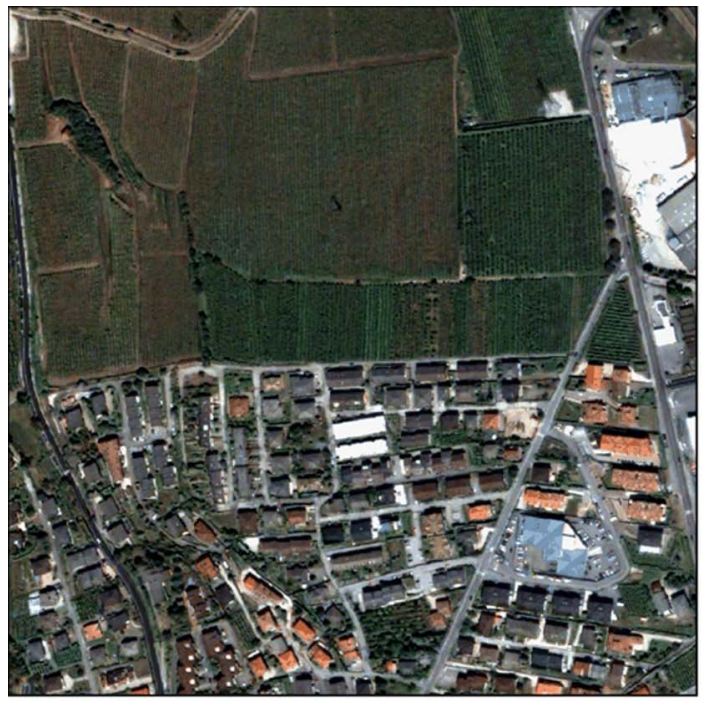

(a)

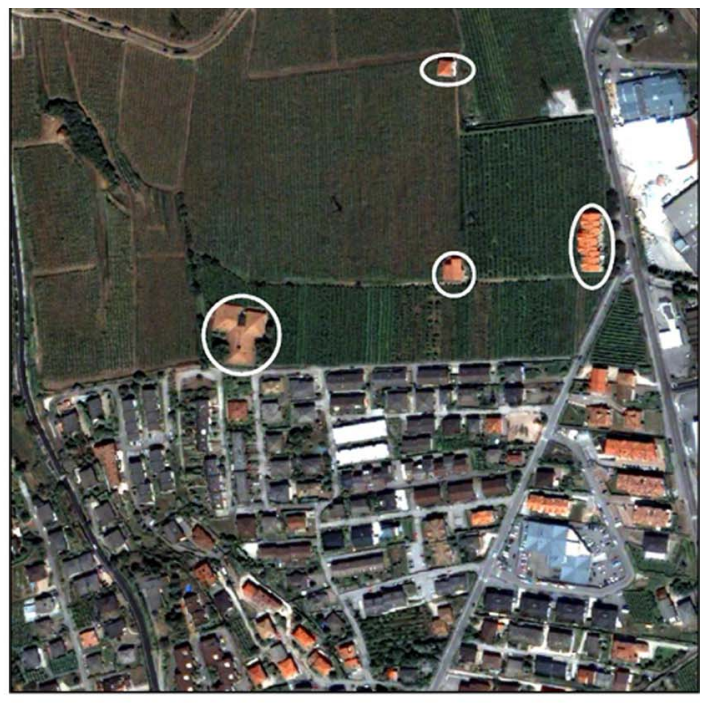

(b)

Fig. 2. True color composition of pansharpened image of the city of Trento (Italy) acquired by the Quickbird VHR multispectral sensor in July 2006 (a) Original image without simulated changes. (b) Original image with simulated changes (pointed out with white circles).

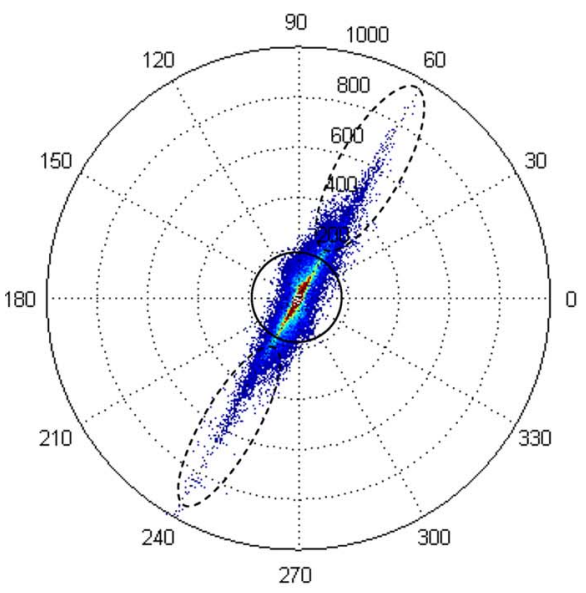

(a)

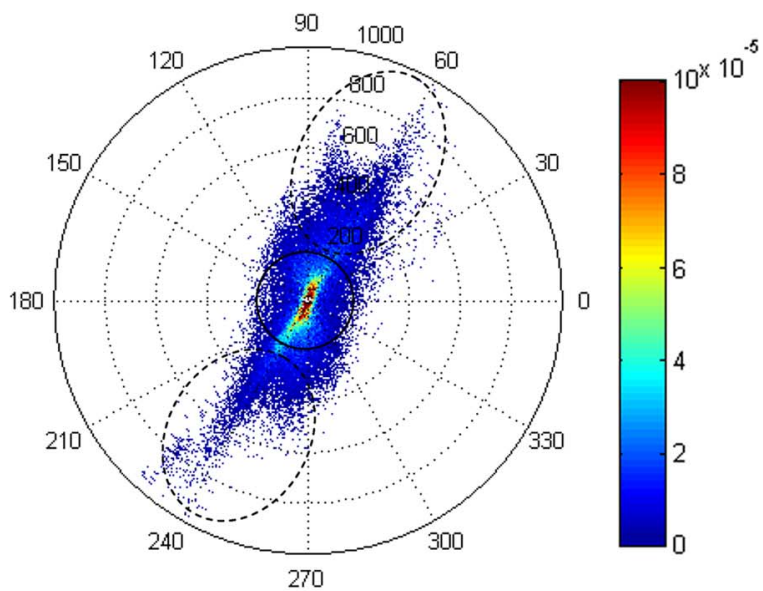

(b)

Fig. 3. Scatterograms in the polar coordinate system obtained by applying CVA to the simulated multitemporal data sets (which do not contain any change) that show (a) 2 pixels and (b) 6 pixels of residual misregistration (Experiment 1).

buildings have similar spectral signatures and are located on agricultural fields. Therefore, the solution to the simulated change-detection problem requires the identification of a single class of changed pixels $\left(\omega_{\mathrm{c}_{1}}\right)$. As in the first experiment, from the simulated image six new images have been generated introducing some pixels of residual misregistration. This resulted in seven multitemporal data sets made up of the original image $\left(\mathbf{X}_{1}\right)$ and one of the simulated images $\left(\mathbf{X}_{2}\right)$. In particular, the two images in the first data set are perfectly aligned and differ only for the simulated changes, while the images in the other data sets show also a residual misregistration between 1 and 6 pixels. It is worth noting that when the images are perfectly coregistered, the application of the CVA technique to $\mathbf{X}_{1}$ and to the image obtained introducing simulated changes leads to a multispectral difference image made up of SCVs with nonzero values only for the simulated changes. Other nonzero SCVs (associated with RN) appear if we compute the scatterograms of pair of misregistered images. Fig. 4 shows an example of the behaviors of such scatterograms obtained by applying the CVA technique to the image $\mathbf{X}_{1}$ and: 1) the simulated image perfectly aligned; 2 ) the simulated image with 2 pixels of residual misregistration; and 3) the simulated image with 6 pixels of residual misregistration. An analysis of these scatterograms (and of the others obtained for different values of misregistration) allowed us to derive the effects of the RN on the class of changed pixels (see Section IV).

\section{Experiment 3: Effects of Misregistration at Different Scales}

Further data sets have been generated from the considered image $\left(\mathbf{X}_{1}\right)$ and the simulated image including changes with a four-pixel misregistration $\left(\mathbf{X}_{2}\right)$ by applying to them a decomposition filter. In this manner, two sets of images $\left(\mathbf{X}_{1}^{n}\right.$ and $\left.\mathbf{X}_{2}^{n}, n=1,2, \ldots, N\right)$ have been generated that have lower scale (resolution) than the original ones. These images show a consistent decrease in detail content. In order to obtain the multiscale representation of the images, in the experimental phase, different decomposition approaches have been used, 


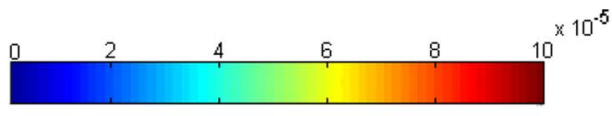

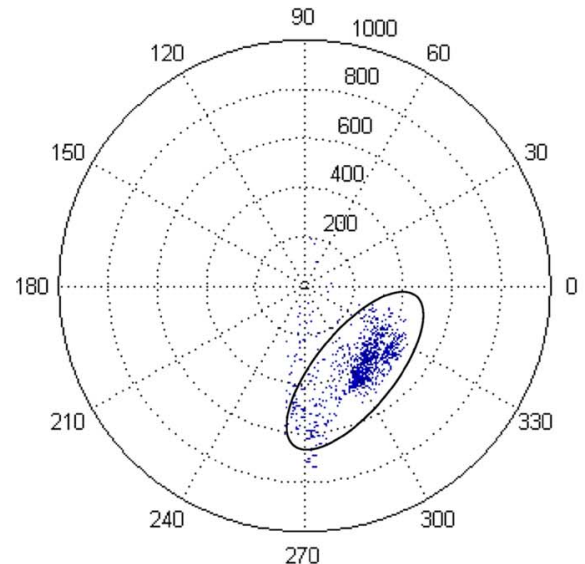

(a)

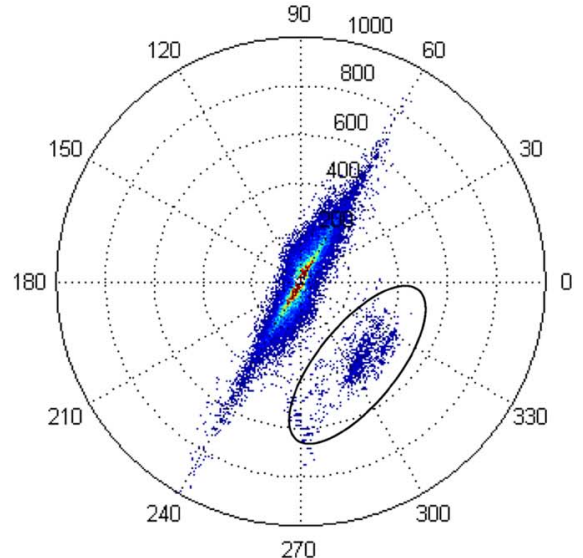

(b)

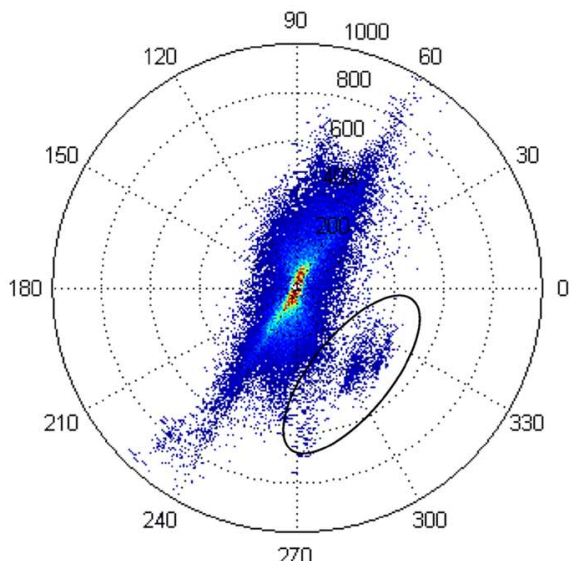

(c)

Fig. 4. Scatterograms in the polar coordinate system obtained by applying CVA to the simulated data sets containing changes in the case of (a) perfect alignment between images, (b) 2 pixels of residual misregistration, and (c) 6 pixels of residual misregistration (Experiment 2).

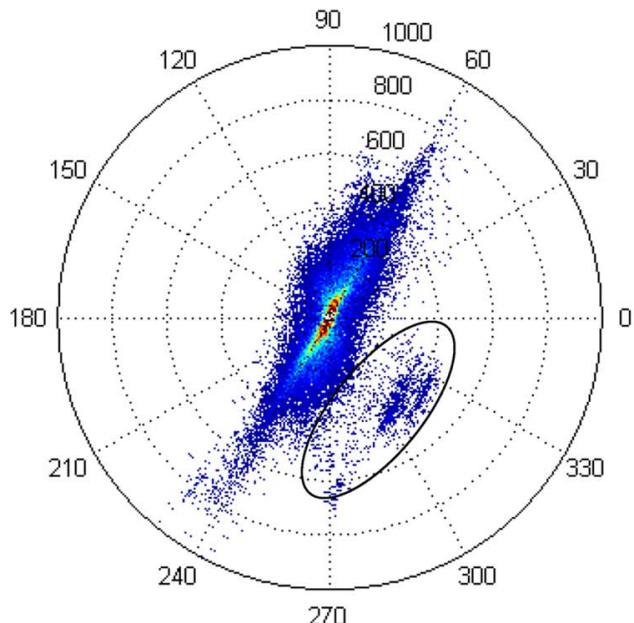

(a)

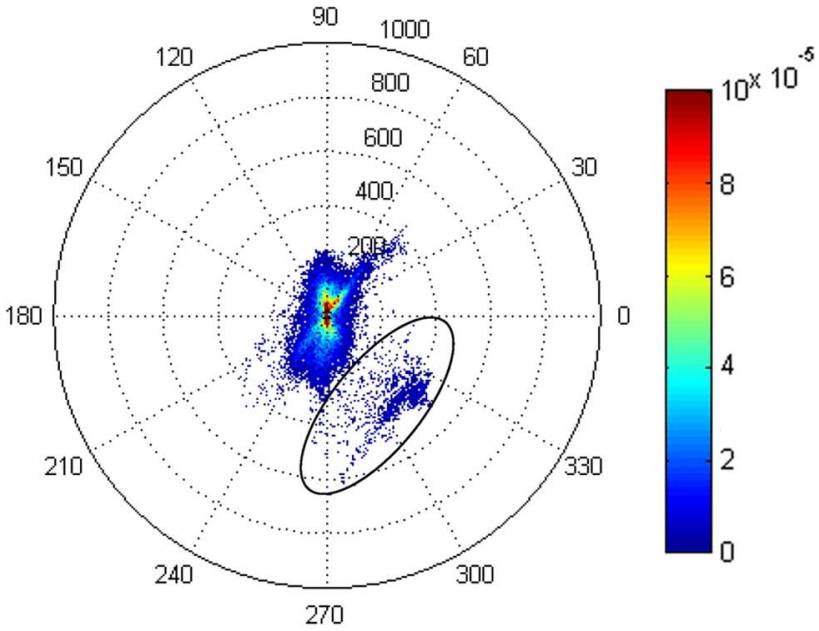

(b)

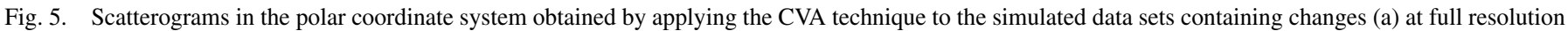
and (b) at a lower scale (level three) (Experiment 3).

such as Laplacian/Gaussian pyramid decomposition, iterative sliding window low-pass filter, recursively upsampled bicubic filter, and wavelet transform. All these approaches provided similar results. For this reason, we report only the analysis obtained by applying to $\mathbf{X}_{1}$ and $\mathbf{X}_{2}$ the Daubechies- 4 stationary wavelet transform (SWT) [15], [16]. In the following, as an example, the results achieved considering the pair of images obtained at the third decomposition level $(n=3)$ are reported. It is worth noting that the choice of the level of decomposition is strictly data and application dependent (see Section VI for details). Fig. 5 shows the scatterograms obtained by applying the CVA technique to images $\mathbf{X}_{1}$ and $\mathbf{X}_{2}$ (full resolution) and to $\mathbf{X}_{1}^{3}$ and $\mathbf{X}_{2}^{3}$, respectively. By comparing these scatterograms (and the others obtained for different values of misregistration and at different resolution levels, which are not reported for space constraints), it is possible to study the effects of multiscale decomposition on the distribution of RN and of real changes (see Section IV).

\section{Properties of RN IN VHR IMAGeS}

An analysis of the scatterograms obtained from the three sets of previously described experiments, and a study on the behavior of SCVs in the polar domain for each investigation setup allowed us to derive some important properties of the RN on both unchanged and changed pixels.

Property 1: RN affects unchanged pixels by: 1) increasing the spread of the cluster in the circle of no-changed pixels $C_{\mathrm{n}}$ with respect to the case of perfectly aligned images; 2) generating clusters of dominant $R N$ in the annulus of changed pixel $A_{\mathrm{c}}$ that have properties very similar to those of changes pixels.

Experiment 1 makes it possible the study of the behavior of the distribution of RN (associated with the distribution of SCVs) versus different amounts of misregistration in the polar domain. As the misalignment increases, the number of multitemporal pixels having the same coordinates but that do not 


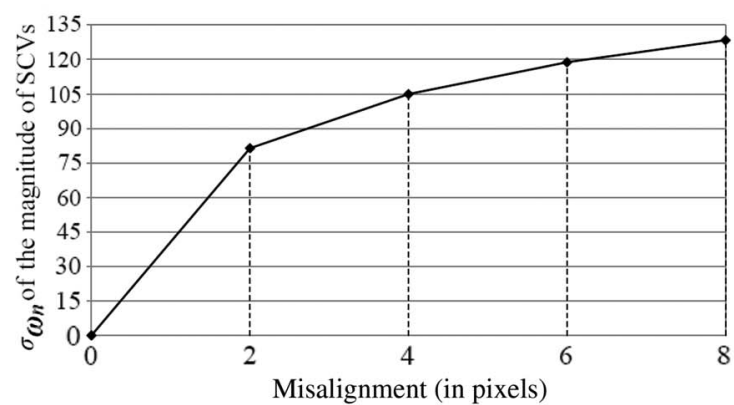

(a)

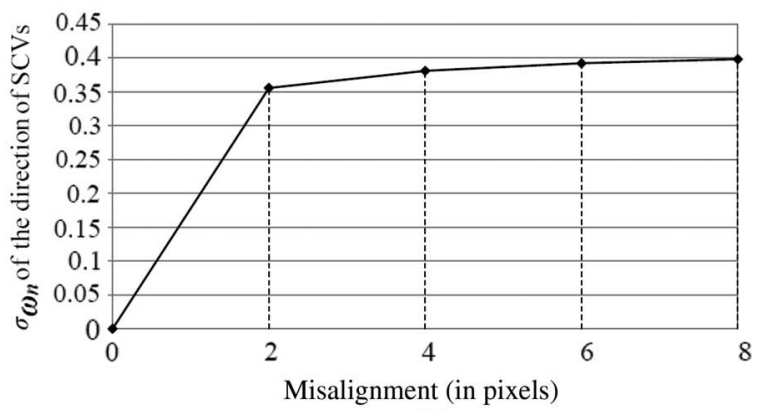

(b)

Fig. 6. Behaviors of the standard deviation of (a) the magnitude and (b) the direction of the SCVs in the cluster of unchanged pixels versus the number of pixels of misalignment (Experiment 1).

correspond to the same position on the ground at the two dates increases. Therefore, the CVA technique performs a comparison between pixels that are not associated to the same area on the ground due to the misalignment. This results in two different contributions to the distribution of $\mathrm{RN}$ in the polar domain: 1) the first one is related to the comparison of pixels that belong to the same object in the two images, but that are not associated with the same position on the ground due to misregistration (slightly different spectral signatures due to the heterogeneity of objects in VHR images) and 2) the second one comes from the comparison between pixels that belong to different objects in the two images (pixels associated with details and border regions). These contributions result in: 1) an increase of the standard deviation of the cluster of unchanged pixels when RN increases and 2) the generation of clusters of unchanged pixels with properties very similar to those of real changes.

Subproperty 1.a: The spread of the cluster in $C_{\mathrm{n}}$ increases by increasing the misalignment.

Let us consider, at first, only the effect of the spectral differences between misaligned pixels of the same object. This effect can be observed in the scatterograms of Fig. 3, where some SCVs associated with unchanged pixels that should stay in $C_{\mathrm{n}}$ fall in $A_{\mathrm{c}}$. Nevertheless, they still show a relative low magnitude and a rather uniform distribution along the direction variable, as it happens for medium resolution images [14] (see regions marked with the continuous line circle in Fig. 3). We can observe that the spread of the cluster of unchanged pixels increases, exhibiting an effect that is sharply amplified with respect to medium resolution images, due to the higher spectral heterogeneity within the objects. It is worth noting that the rather uniform distribution of SCVs along the direction is due to the fact that the structures of objects are usually different for different elements in the scene.

A quantitative analysis carried out on both the magnitude and the direction of SCVs shows that the standard deviation $\sigma_{\omega_{\mathrm{n}}}$ of the class of unchanged patterns $\omega_{n}$ increases in a nonlinear way by increasing the misalignment (see Fig. 6) and, as expected, it tends to saturate when the residual $\mathrm{RN}$ is over a given threshold.

Statistically, as reported in [14] for the class of unchanged pixels, RN generated by the comparison of pixels that belong to the same object can be modeled as a mixture of Gaussian distributions with the same mean values (as the distributions at the two dates are related to the same class) in the Cartesian domain, which corresponds to a Rayleigh distribution along the magnitude variable of the polar domain and to a uniform distribution along the direction variable.

Subproperty 1.b: The clusters of dominant $R N$ in $A_{\mathrm{c}}$ have properties very similar to those of real changes and are made up of a number of patterns that increases by increasing the misalignment.

Let us now consider the effects of pixels that at the two acquisition dates belong to different objects on the ground. In this case, significantly different spectral signatures are compared leading to SCVs with large magnitude values. This behavior can be observed in the scatterograms of Fig. 3 where it is possible to note that a large number of unchanged SCVs show a magnitude significantly higher than expected, thus falling in $A_{\mathrm{c}}$ (see regions marked with dashed circles in Fig. 3). In the medium resolution case, the distribution of such SCVs is nearly uniform along the direction [14]. On the contrary, when dealing with VHR images, their distribution has preferential directions, resulting in clusters of pixels of $\mathrm{RN}$ in $A_{\mathrm{c}}$ that exhibit properties very similar to those of changed pixels. Such an effect is mainly due to the comparison of misaligned pixels belonging to different objects with similar structures in different positions of the images. This can be explained, for example, with the regular structure of the urban areas and of the crop rows, as well as with the high-frequency content of the VHR images. The number of SCVs composing these clusters increases by increasing the amount of RN. It is worth noting that, on the contrary, when dealing with medium resolution images, the number of misregistered pixels belonging to different objects is small, and the effects of RN are less evident and more uniformly distributed along the direction variable. This is due to both the small amount of geometrical details contained in such images, and the intrinsic effectiveness of classic registration algorithms on medium resolution data. We define the annular sectors in the polar domain associated with these clusters as sectors of dominant $R N S_{\mathrm{RN}_{i}}^{\mathrm{D}}$

$$
S_{\mathrm{RN}_{i}}^{\mathrm{D}}=\left\{\rho, \vartheta: \rho \geq T \text { and } \vartheta_{i_{1}} \leq \vartheta \leq \vartheta_{i_{2}}, 0 \leq \vartheta_{i_{1}}<\vartheta_{i_{2}}<2 \pi\right\} .
$$

Each $S_{\mathrm{RN}_{i}}^{\mathrm{D}}$ can be represented in the polar domain as a sector within $A_{\mathrm{c}}$ bounded from two angular thresholds $\vartheta_{i_{1}}$ and $\vartheta_{i_{2}}$. This is not surprising as SCVs due to misregistration, exactly as SCVs of true changes, are originated from the comparison of pixels that are associated with different objects on the ground at the two acquisition dates. It follows that sectors of dominant RN 


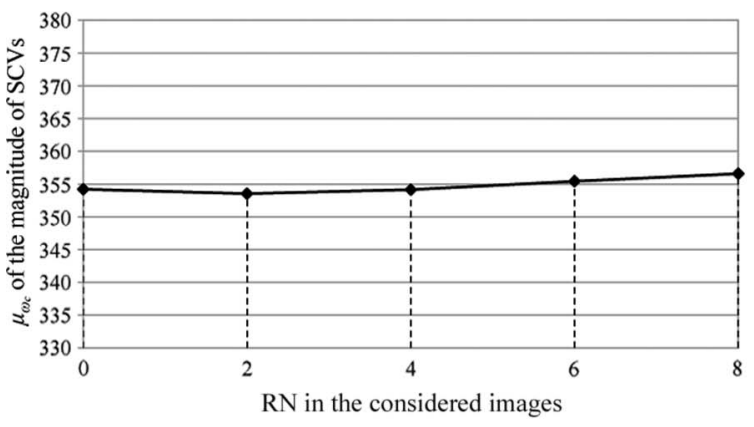

(a)

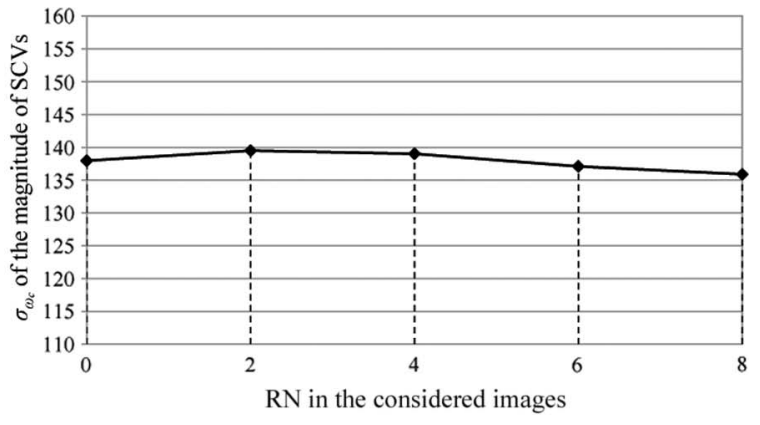

(b)

Fig. 7. Behaviors of (a) the mean value $\mu_{\omega_{\mathrm{c}}}$ and (b) the standard deviation $\sigma_{\omega_{\mathrm{c}}}$ of the magnitude of SCVs in the cluster of changed pixels versus the number of pixels of misalignment (Experiment 2).

are very critical because at full resolution, they cannot be distinguished from sectors of true changes, resulting in a significant false alarm rate in the change-detection process. Statistically, as reported in [14] for the class of changed pixels, RN generated by the comparison of pixels that belong to different classes can be modeled as a mixture of Gaussian distributions with different mean values in the Cartesian domain which corresponds to a Ricean distribution along the magnitude variable of the polar domain and to a nonuniform distribution along the direction variable.

Property 2: Statistical properties of clusters associated with changed pixels in $A_{\mathrm{c}}$ slowly vary with the amount of misalignment.

Experiment 2 points out the behaviors of SCVs associated with changed pixels versus the amount of misalignment that affects the considered simulated data sets. Observing Fig. 4, it is possible to note that SCVs associated with the class of changed pixels $\omega_{\mathrm{c}_{1}}$ are not significantly affected by an increase of the amount of misregistration between images. Indeed, the cluster of changed pixels can be easily identified in all the three scatterograms and shows quite stable behaviors (see regions marked with circles in Fig. 4). The position of the annular sector $S_{1}$ (which identifies pixels belonging to $\omega_{\mathrm{c}_{1}}$ ) is almost invariant with the misregistration. This behavior allows one to conclude that the RN does not affect significantly the properties of the cluster of changed pixels. This is confirmed from a quantitative analysis of the behavior of the mean value $\mu_{\omega_{c}}$ and standard deviation $\sigma_{\omega_{c}}$ of the magnitude of SCVs in the cluster of changed pixels $\omega_{\mathrm{c}}$ (for simplicity of notation in the following $\omega_{\mathrm{c}_{1}}$ will be indicated as $\omega_{\mathrm{c}}$ ) versus the amount of misregistration (in pixels). As shown from Fig. 7, these behaviors do not show significant variations by increasing misregistration.

Nonetheless, the RN indirectly affects the detection of changed pixels (see Property 1) as: 1) the overlap between clusters of changed and unchanged pixels increases when the standard deviation of the patterns in $C_{\mathrm{n}}$ increases and 2) the presence of sectors of dominant $\mathrm{RN}$ in $A_{\mathrm{c}}$ results in false alarms.

Property 3: Clusters of dominant $R N$ in $A_{\mathrm{c}}$ exhibit significant variations of properties versus the scale (resolution) of the images.

From experiment 3 , we can observe the effects of a multiscale decomposition of the images on pixels associated with both changed and unchanged areas. Let us first consider only un-

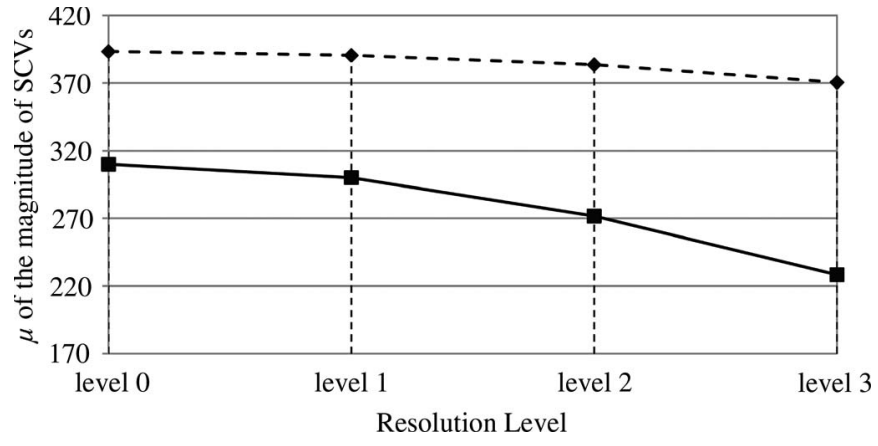

Fig. 8. Behavior of the mean value of the magnitude of SCVs versus the resolution levels (scale) for clusters (dashed line) of change and (continuous line) of RN.

changed pixels (changed pixels will be discussed in property 4). As the resolution of the images decreases, the presence of small and thin structures diminishes. This results in a reduced impact of $\mathrm{RN}$ at lower scales (resolutions) as the details and border regions are smoothed out from the low-pass effects associated with scale reduction. Comparing the scatterograms of Fig. 5 (derived from experiment 3), it can be observed that reducing the scale, SCVs associated with RN tend to disappear. In other words, decreasing the resolution sectors of dominant $\mathrm{RN}$ tend to disappear, thus exhibiting a nonstationary behavior with respect to the scale. In particular, such SCV s tend to collapse within $C_{\mathrm{n}}$. This is confirmed from Fig. 8, which reports the behavior of the mean value of the magnitude of SCVs associated with RN versus the resolution levels (scales). As can be seen from the continuous line in the diagram, the mean value of $\mathrm{RN}$ clusters rapidly decreases by reducing the resolution. This property is very important in the definition of a strategy for estimating the distribution of RN in VHR images (see Section V).

Property 4: Clusters associated with changed pixels in $A_{\mathrm{c}}$ exhibit slow varying statistical properties versus the scale (resolution) of the images.

From experiment 3 , it is also possible to observe the behavior of the cluster of changed pixels when the scale decreases. Observing regions marked with circles in Fig. 5, it is possible to note that the cluster of pixels associated with true changes reduces its spread, but it is not completely smoothed out when the resolution decreases. In other words, it shows a nearby stationary behavior versus the resolution. This is confirmed by an analysis of the behavior of the mean value of the magnitude 
of SCVs associated with true changes versus the scale. As it can be seen from the dashed line in Fig. 8, the mean value slightly varies with the resolution, but it decreases slower than the one of SCVs associated with RN (continuous line in Fig. 8).

From properties 3 and 4, it follows that the behaviors of changed and unchanged (i.e., the ones due to RN) SCVs that fall in $A_{\mathrm{c}}$ versus the resolution are different: decreasing the resolution, sectors of changes, unlike sectors of dominant $\mathrm{RN}$, are preserved. It is worth noting that this property is true under the reasonable and realistic assumption that given the very high geometrical resolution of the sensor, the true significant changes are associated with objects with a nonnegligible size. This results in an intrinsic robustness of changes to the scale. On the contrary, misregistration appears in the difference image with linear (or nonlinear) and relatively thin structures having different orientations that are smoothed out from the scale reduction process. Properties 3 and 4 can be exploited for defining an adaptive strategy for estimating the statistical distribution of RN. This strategy is described in the next section.

\section{Proposed Technique for the Adaptive ESTIMATION OF THE RN DISTRIBUTION}

The properties of $\mathrm{RN}$ described in the previous section suggest us to exploit the behaviors of SCVs in the polar domain at different resolution levels (scales) for explicitly estimating the statistical distribution of RN. In particular, properties 3 and 4 clearly show the usefulness of a multiresolution decomposition in identifying and separating annular sectors of dominant RN from annular sectors of real changes. Given the very high geometrical resolution of images, we assume that true significant changes are associated with objects with a nonnegligible size. On the contrary, misregistration appears in the difference image with linear (or nonlinear) and relatively thin structures having different orientations. Therefore, if we reduce the resolution of images, we implicitly decrease the impact of the $\mathrm{RN}$ with respect to that on the original scene (Property 3), while true changes maintain a good stability (Property 4). In other words, the lower is the geometrical resolution, the lower is the probability of identifying in the polar representation annular sectors of dominant RN. This means that at low resolution, in the annulus of changed pixels, mainly, sectors (i.e., clusters) due to the presence of true changes on the ground are detected. Thus, by comparing the clusters present in the polar domain at full resolution and at reduced resolution, it is possible to identify annular sectors dominated from $\mathrm{RN}$ and separate them from annular sectors of changes. It is worth noting that this is made possible from the thin structures associated with $\mathrm{RN}$ that result in strong changes in the corresponding SCV clusters when the low-pass effect of the scale reduction is considered.

On the basis of the aforementioned analysis, we propose an adaptive multiscale strategy that exploits the behaviors of SCVs to identify the distribution of the RN. The proposed technique compares the distribution of the SCVs at the highest resolution level with the one at a lower level in order to derive the distribution of RN at full resolution. In particular, first of all the two multitemporal images are decomposed according to a multiscale transformation (as described in Section III different algorithms can be used, like SWT, recursively upsampled bicubic filter, etc.). In greater detail, we applied the 2-D discrete SWT; this decomposition technique is obtained as an extension of the 1-D discrete SWT by applying 1-D filters independently along both dimensions of the considered image. In particular, two filters with different impulse responses are considered to built up the SWT filter bank: 1) a lowpass filter with impulse response $l($.$) and 2) a high-pass filter$ with impulse response $h($.$) . A one-step wavelet decomposition$ applies both filters separately: first along columns and then along rows. The original image $\mathbf{X}_{i}(i=1,2)$ is decomposed into a low-resolution image (the approximation subband $\mathbf{X}_{i}^{L L}$ ), containing low spatial frequencies in both the horizontal and the vertical direction, and three detail images $\mathbf{X}_{i}^{L H}, \mathbf{X}_{i}^{H L}$, and $\mathbf{X}_{i}^{H H}$, which correspond to the horizontal, vertical and diagonal detail subbands at resolution level 1, respectively. Note that, superscripts $L L, L H, H L$, and $H H$ specify the order on which high- and low-pass filters have been applied to obtain the considered subband. The multiscale decomposition is obtained by recursively applying the described procedure to the approximation subband obtained at each scale $2^{n}$. Thus, the output at a generic resolution level $n$ can be express analytically as follows:

$$
\begin{aligned}
& \mathbf{X}_{i}^{L L(n+1)}(i, j)=\sum_{p=0}^{D^{n}-1} \sum_{q=0}^{D^{n}-1} l^{n}[p] l^{n}[q] \mathbf{X}_{i}^{L L n}(i+p, j+q) \\
& \mathbf{X}_{i}^{L H(n+1)}(i, j)=\sum_{p=0}^{D^{n}-1} \sum_{q=0}^{D^{n}-1} l^{n}[p] h^{n}[q] \mathbf{X}_{i}^{L L n}(i+p, j+q) \\
& \mathbf{X}_{i}^{H L(n+1)}(i, j)=\sum_{p=0}^{D^{n}-1} \sum_{q=0}^{D^{n}-1} h^{n}[p] l^{n}[q] \mathbf{X}_{i}^{L L n}(i+p, j+q) \\
& \mathbf{X}_{i}^{H H(n+1)}(i, j)=\sum_{p=0}^{D^{n}-1} \sum_{q=0}^{D^{n}-1} h^{n}[p] h^{n}[q] \mathbf{X}_{i}^{L L n}(i+p, j+q)
\end{aligned}
$$

where $D^{n}$ is the length of the wavelet filters at resolution level $n$. At each decomposition step, the length of the impulse response of both high- and low-pass filters is upsampled by a factor of two. Thus, filter coefficients for computing subbands at resolution level $n+1$ can be obtained by applying a dilation operation to the filter coefficients used to compute level $n$. In particular, $2^{n-1}$ zeros are inserted between the filter coefficients used to compute subbands at the lower resolution level. This allows a reduction in the bandwidth of the filters by a factor two between subsequent resolution levels. Filter coefficients of the first decomposition step for $n=0$ depend on the selected wavelet family and on the length of the chosen wavelet filter. To this purpose, we selected the Daubechies wavelet family and set the filter length to eight. The finite impulse response of the high-pass filter for the decomposition step is obtained by satisfying the properties of the quadrature mirror filters. This is done by reversing the order of the low-pass decomposition filter coefficients and by changing the sign of the even indexed coefficients [16].

In order to perform the proposed analysis, one must return to the original image domain. This is done by applying only 
to the approximation subbands the 2-D inverse discrete SWT at each resolution level independently. In this way, we obtain two sets of images $\mathbf{X}_{\mathrm{MS}_{i}}=\left\{\mathbf{X}_{i}^{0}, \ldots, \mathbf{X}_{i}^{n}, \ldots, \mathbf{X}_{i}^{N-1}\right\}$ where the subscript $i(i=1,2)$ denotes the acquisition date, and the superscript $n(n=0,1, \ldots, N-1)$ indicates the resolution level (note that $\mathbf{X}_{i}^{0} \equiv \mathbf{X}_{i}$ ). Then, the CVA technique is applied to each corresponding pair of images $\left(\mathbf{X}_{1}^{n}, \mathbf{X}_{2}^{n}\right)$, and the distributions of the direction of SCVs at different resolution levels are analyzed. In particular, the behaviors of SCVs in $A_{\mathrm{c}}$ are studied. To this purpose, we compute the conditional density of the direction of pixels in $A_{\mathrm{c}}$. In order to estimate this distribution, we take advantages from the Parzen windows technique [17]-[20], which is a basic and effective estimation method for one-dimension problems. According to this technique, the density estimation can be computed as

$$
\hat{p}_{\mathrm{n}}(\vartheta \mid \rho \geq T)=\frac{1}{M_{\mathrm{n}}} \sum_{m=1}^{M_{\mathrm{n}}} \frac{1}{h_{\mathrm{n}}} \gamma\left(\frac{\vartheta-\vartheta_{m}}{h_{\mathrm{n}}}\right)
$$

where $T$ is the threshold value that separates the circle of no-changed pixels from the annulus of changed pixels (it can be retrieved either manually or in an automatic way through one of the algorithms proposed in the literature [21], [22], see Section II), $n(n=0,1, \ldots, N-1)$ denotes the resolution level at which the estimation is computed, $\vartheta_{m}$ represents the direction value of the $m$ th SCV in $A_{\mathrm{c}}, M_{\mathrm{n}}$ is the number of SCVs in $A_{\mathrm{c}}$ at scale $n, \gamma($.$) is the kernel function used in the$ estimation process, and $h_{\mathrm{n}}$ is the width of the kernel window (smoothing parameter) at scale $n$.

In particular, we used Gaussian kernel, so that the final estimation is given by

$$
\hat{p}_{\mathrm{n}}(\vartheta \mid \rho \geq T)=\frac{1}{M_{\mathrm{n}}} \sum_{m=1}^{M_{\mathrm{n}}} \frac{1}{h_{\mathrm{n}} \sqrt{2 \pi}} \exp \left[-\frac{1}{2}\left(\frac{\vartheta-\vartheta_{m}}{h_{\mathrm{n}}}\right)^{2}\right] .
$$

For what concerns the smoothing parameter, which, in our case, is represented by the standard deviation of the Gaussian function, we propose to compute it as a function of the number of pixels that fall in $A_{\mathrm{c}}$. In particular, considering a Gaussian kernel, the width value at scale $n$ can be derived as in [17]

$$
h_{\mathrm{n}}=\operatorname{sig} *\left(\frac{4}{3 M_{\mathrm{n}}}\right)^{1 / 5}
$$

where

$$
\operatorname{sig}=\underset{m=1, \ldots, M_{\mathrm{n}}}{\operatorname{median}}\left|\vartheta_{m}-\underset{m=1, \ldots, M_{\mathrm{n}}}{\operatorname{median}}\left(\vartheta_{m}\right)\right| / 0.6745 .
$$

Then, we observe the behaviors of $\hat{p}_{\mathrm{n}}(\vartheta \mid \rho \geq T)$ versus the scale. According to the properties of RN, this density decreases at reduced resolutions in the annular sectors of dominant RN $S_{\mathrm{RN}_{i}}^{\mathrm{D}}$, whereas it remains nearby constant in the annular sectors of true changes $S_{k}$. On the basis of this analysis, we propose to estimate the conditional density of $\mathrm{RN}$ in the direction domain $\hat{p}_{\mathrm{RN}}(\vartheta \mid \rho \geq T)$ as

$$
\begin{aligned}
\hat{p}_{\mathrm{RN}}(\vartheta \mid \rho \geq T)=C[ & P_{0}(\rho \geq T) \hat{p}_{0}(\vartheta \mid \rho \geq T) \\
& \left.-P_{N-1}(\rho \geq T) \hat{p}_{N-1}(\vartheta \mid \rho \geq T)\right]
\end{aligned}
$$

where $P_{\mathrm{n}}(\rho \geq T)$ is the probability of SCVs to be in $A_{\mathrm{c}}$ at scale $n, \hat{p}_{0}(\vartheta \mid \rho \geq T)$ and $\hat{p}_{N-1}(\vartheta \mid \rho \geq T)$ are the marginal conditional densities of the direction of pixels in $A_{\mathrm{c}}$ at the full resolution and at the lowest considered resolution level $(N-1)$, respectively, and $C$ is a constant defined such that $\int_{-\infty}^{+\infty} \hat{p}_{\mathrm{RN}}(\vartheta \mid \rho \geq T) \mathrm{d} \vartheta=1$. The term $P_{\mathrm{n}}(\rho \geq T)$ in (13) is necessary in order to obtain a reliable comparison between distributions at different resolution levels.

In this way, we obtain an explicit estimation of the distribution of RN that is adaptive (in the sense that it intrinsically takes into account the properties of the considered images). It is worth noting that this estimated distribution represents the behavior of RN at full scale (resolution). In the proposed technique, the analysis at the lowest resolution is only used for separating the RN contribution from that of true changes (and of other possible sources of noise). The sensitivity of the estimation depends on the lowest level $N-1$ of decomposition considered. The lower the level is, the greater the sensitivity of the estimated distribution to the minor components of RN is. Nonetheless, considering applications like change detection, it is important to choose the lowest scale according to the smallest size of expected changes, as they must be preserved in the degraded image in order to be detected.

\section{EXPERIMENTAL RESULTS}

This section presents an experimental analysis on both the reliability of the derived properties of $\mathrm{RN}$ and the effectiveness of the proposed method to estimate the distribution of $\mathrm{RN}$ on real multitemporal images. The investigation was conducted on two different test areas of a pair of Quickbird images acquired on the Trento city (Italy) in October 2005 and July 2006. For both test areas, the final data set is made up of two pansharpened radiometrically corrected multitemporal and multispectral images of $984 \times 984$ pixels, with a geometrical resolution of $0.7 \mathrm{~m}$, which after preprocessing show a residual misregistration of about 1 pixel on ground control points. ${ }^{2}$

For applying the proposed method to the estimation of $\mathrm{RN}$, the original images $\mathbf{X}_{1}$ and $\mathbf{X}_{2}$ were transformed to lower scales through a four-step SWT [15], [24] using fourth-order orthogonal filters of the Daubechies family. The maximum level of decomposition was selected according to a tradeoff between the degree of sensitivity desired in the RN estimation and the size of the expected main change structures present in the images. Then, the CVA technique was applied to the images at different scales. In order to separate the circle of no-changed pixels $\left(C_{\mathrm{n}}\right)$ from the annulus of changed pixels $\left(A_{\mathrm{c}}\right)$, for each data set, a proper threshold value $T$ on the magnitude variable was retrieved according to a trial-and-error procedure. (We did not use an automatic technique for avoiding biases introduced from the threshold selection method in the evaluation of the effectiveness of the proposed method. However, at an operational level, one of the thresholding algorithms proposed in the

\footnotetext{
${ }^{2}$ It is worth noting that we carried out all the analysis using pansharpened images, as we expect that the pansharpening process can emphasized the effects of misregistration. However, similar results can be obtained on original multispectral images at lower resolution $(2.4 \mathrm{~m})$. We refer the reader to [23] for greater details on the effects of pansharpening on change detection.
} 


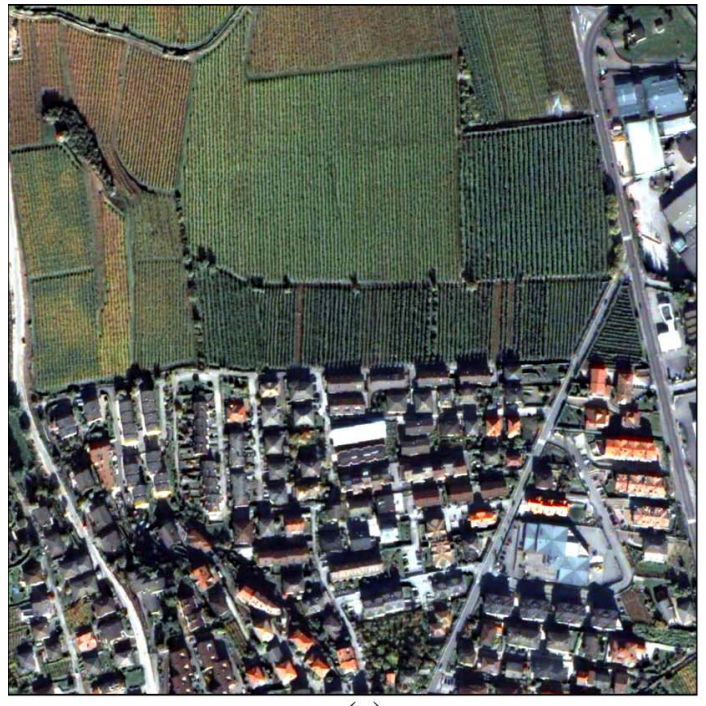

(a)

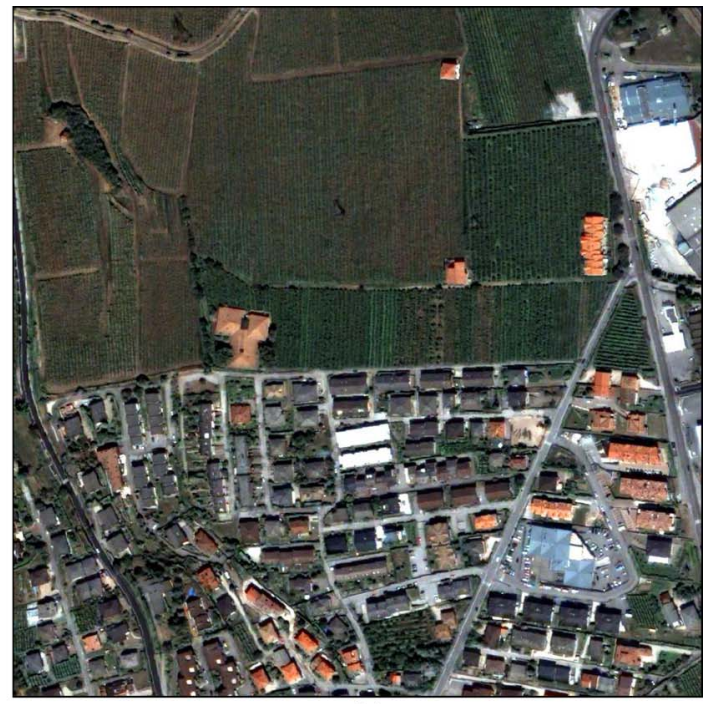

(b)

Fig. 9. True color composition of pansharpened images of the Trento city (Italy) acquired by the Quickbird VHR multispectral sensor in (a) October 2005 and (b) July 2006 (Test site 1).

literature can be used [21], [22].) In greater detail, in order to find the optimal threshold for our purposes, the whole analysis for the estimation of the RN distribution has been conducted for different values of the threshold $T$ in a consistent range of the magnitude values. All of them provided similar results in the estimation of RN. For space constraints, in the following, only the results obtained with a single threshold value for each data set are reported. The marginal conditional densities of the directions of pixels in $A_{\mathrm{c}}$ at the highest resolution and at a lower resolution levels were computed according to (10), and finally the conditional density of RN was estimated according to (13). In order to assess the effectiveness of the proposed technique for the identification of the distribution of RN, such a distribution was thresholded, and the direction intervals in $A_{\mathrm{c}}$, where $\hat{p}_{\mathrm{RN}}(\vartheta \mid \rho \geq T)$ was higher than a given threshold, were recognized as dominated from $\mathrm{RN}$. Then, the $\mathrm{RN}$ maps were derived on the basis of the results obtained by thresholding $\hat{p}_{\mathrm{RN}}(\vartheta \mid \rho \geq T)$, and a qualitative analysis was performed in order to assess the effectiveness of the proposed estimation technique.

\section{A. Test Site 1: Urban and Rural Areas}

The first test site considered (see Fig. 9) covers both an urban area and a rural one. Between the two acquisitions, two kinds of changes occurred: 1) simulated changes (see Section III-B on the procedure adopted to simulate them) that consist of new houses introduced on the rural area and 2) real changes that consist of some roofs rebuilt in the urban area. In order to assess the reliability of the proposed technique, the previously described procedure was applied to the two images. Fig. 10 shows the behaviors of the marginal conditional densities of the direction in $A_{\mathrm{c}}\left[\hat{p}_{\mathrm{n}}(\vartheta \mid \rho \geq T)\right]$ computed according to (10) and corrected by the term $P_{\mathrm{n}}(\rho \geq T)$, after applying the CVA technique to the red and near-infrared spectral channels of: 1) the original images at full resolution (continuous line in Fig. 10) and 2) the low-resolution images yielded at level four of the Daubechies stationary wavelet decomposition (dashed

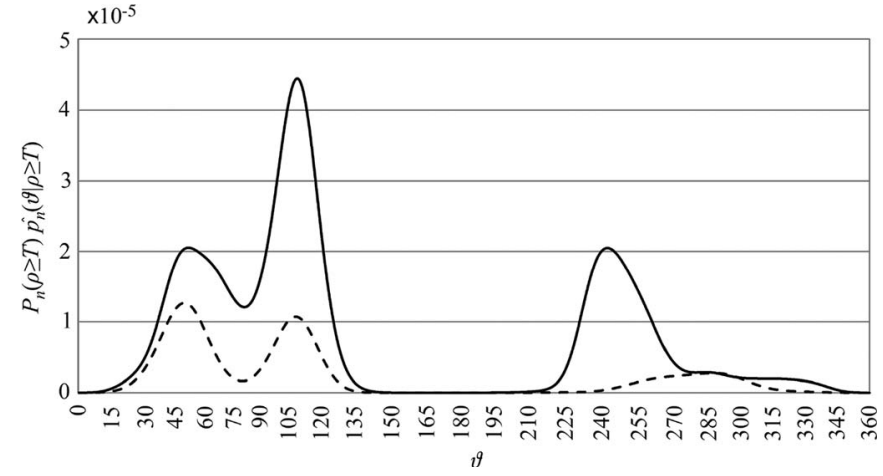

Fig. 10. Marginal weighted conditional densities $P_{\mathrm{n}}(\rho \geq T) \hat{p}_{\mathrm{n}}(\vartheta \mid \rho \geq T)$ of the direction in $A_{\mathrm{c}}$ (continuous line) at full resolution and (dashed line) at level four of the Daubechies SWT (Test site 1).

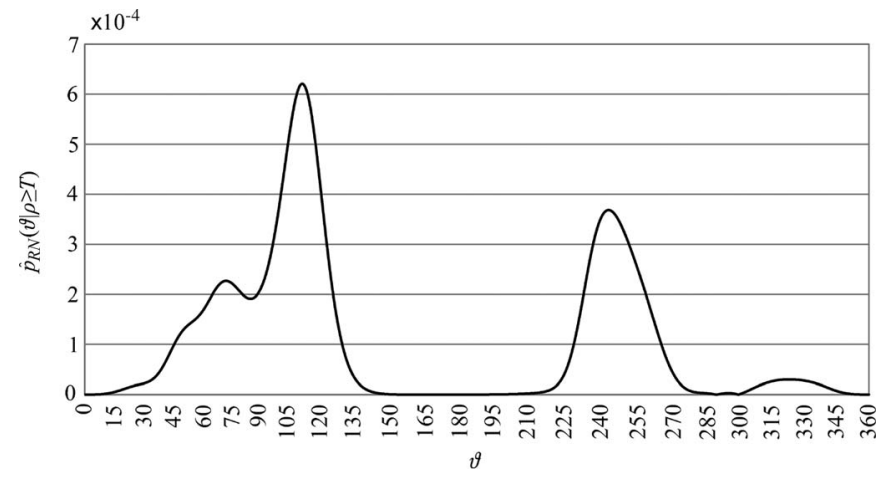

Fig. 11. Estimated conditional density $\hat{p}_{\mathrm{RN}}(\vartheta \mid \rho \geq T)$ of RN obtained with the proposed technique (Test site 1).

line in Fig. 10). As previously mentioned, red and near-infrared spectral channels were considered as they better represent changes occurred between the two dates. The estimation of the marginal conditional density of $\mathrm{RN} \hat{p}_{\mathrm{RN}}(\vartheta \mid \rho \geq T)$ was derived from the two aforementioned densities according to (13) (see Fig. 11). From an analysis of the behavior of $\hat{p}_{\mathrm{RN}}(\vartheta \mid \rho \geq T)$, it is possible to identify three main modes, which potentially define sectors where the RN is dominant. A comparison between 


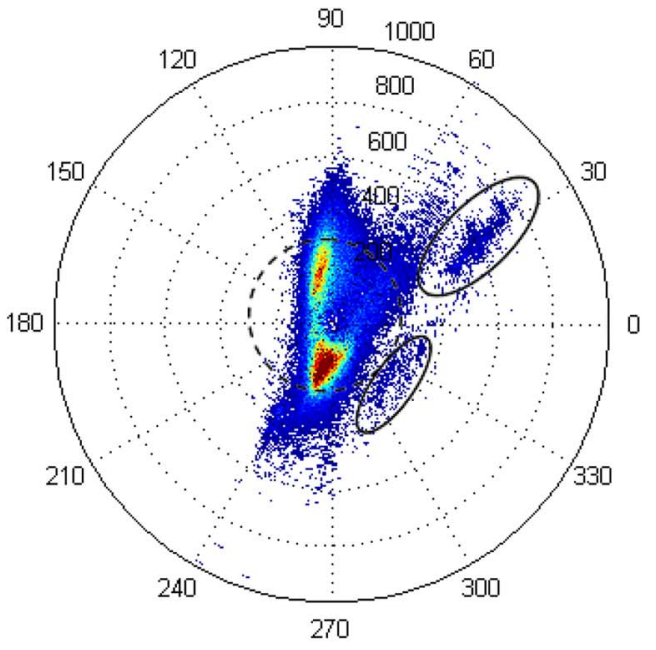

(a)

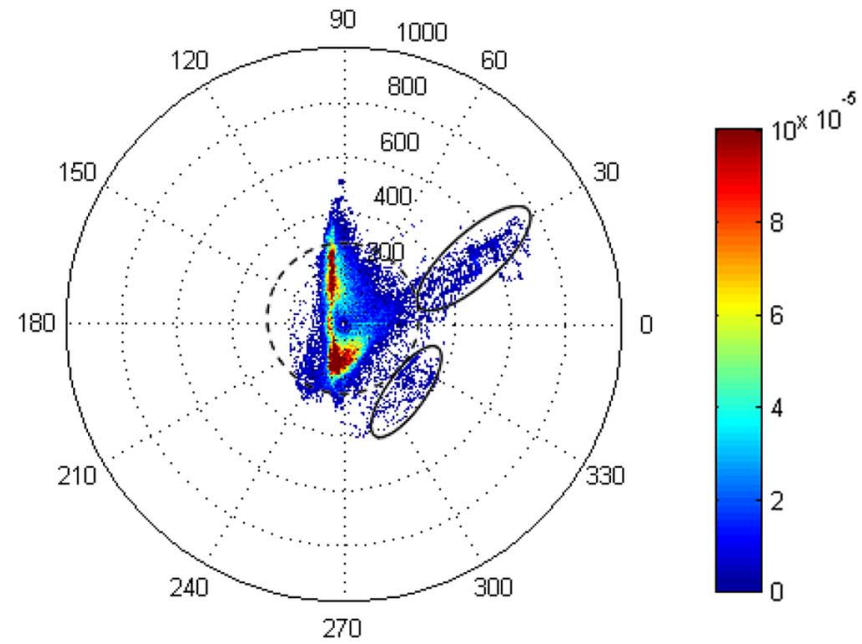

(b)

Fig. 12. Scatterograms in the polar coordinate system of (a) the full-resolution original difference image $\mathbf{X}_{\mathrm{D}}^{0}$ and (b) the low-resolution image $\mathbf{X}_{\mathrm{D}}^{4}$ obtained at level four of the wavelet decomposition (Test site 1). Dashed circles separate $C_{\mathrm{n}}$ from $A_{\mathrm{c}}$, while continuous circles indicate sectors of true changes.

the scatterograms at full and at low resolution (see Fig. 12) points out that in the sectors corresponding to the three modes of $\hat{p}_{\mathrm{RN}}(\vartheta \mid \rho \geq T)$, the density of the magnitude of SCVs in the annulus of changed pixel reduces significantly when the resolution decreases, whereas in the others, it is nearly constant. In particular, it is possible to verify that the sectors in which the behavior of SCVs is quite stable correspond to sectors of true changes (continuous circles in Fig. 12). This behavior also confirms the properties derived from the simulated data sets.

To further understand the effectiveness of the proposed estimation technique, we applied a threshold to $\hat{p}_{\mathrm{RN}}(\vartheta \mid \rho \geq T)$ to identify sectors of dominant RN. The threshold value was empirically fixed equal to $1 \times 10^{-4}$. Thus, in the annulus of changed pixels (defined by applying a threshold $T=310$ to the magnitude of SCVs), the sectors of dominant RN were identified between $35^{\circ}$ and $115^{\circ}$ and between $225^{\circ}$ and $265^{\circ}$. In order to perform a qualitative analysis of the estimation, Fig. 13 shows the map of pixels associated with the estimated sectors of dominant RN. A visual analysis of this map confirms that the regions identified as $\mathrm{RN}$ by the proposed technique are associated with areas that show the effects of misregistration between the multitemporal images, as they mainly refer to border regions of buildings located in the urban area, to roads, and to crop rows. In addition, it is possible to note that the regions identified in the RN map do not belong to areas of changes. This behavior confirms the effectiveness of the proposed technique that properly distinguishes between $\mathrm{RN}$ and true changes contributions in the estimation of $\hat{p}_{\mathrm{RN}}(\vartheta \mid \rho \geq T)$. It is worth noting that the proposed technique marks as RN, the boundaries of the four simulated changes. This happens as, in order to make the changes more realistic, together with the roofs also some pixels surrounding buildings have been copied; these pixels result in changes with thin structure and small size with respect to the sensor resolution. Thus, they do not satisfy the basic assumption that true significant changes are associated with objects with nonnegligible size and, consequently, appear correctly as RN.

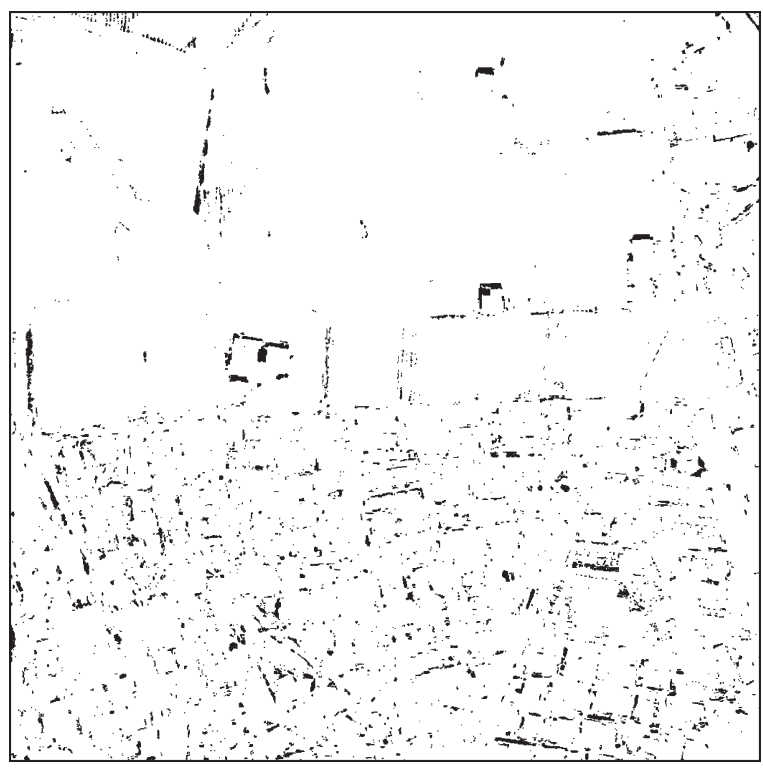

Fig. 13. RN map obtained by thresholding the $\hat{p}_{\mathrm{RN}}(\vartheta \mid \rho \geq T)$ obtained with the proposed technique (Test site 1).

\section{B. Test Site 2: Industrial and Rural Area}

The second test site considered (see Fig. 14) mainly covers an industrial area. Moreover, in this case, two kinds of changes occurred in the two images: 1) natural changes of the land cover in rural areas and along the river bank and 2) man-made changes in the roofs of the industrial area. As for the previous data set, the marginal conditional densities of the direction in $A_{\mathrm{c}}$ were computed according to (10) after applying the CVA technique to the red and near-infrared spectral channels of the original images at full resolution (continuous line in Fig. 15) and the low-resolution images yielded at level four of the Daubechies stationary wavelet decomposition (dashed line in Fig. 15). From these distributions, the $\hat{p}_{\mathrm{RN}}(\vartheta \mid \rho \geq T)$ was estimated according to (13) (Fig. 16). Moreover, in this 


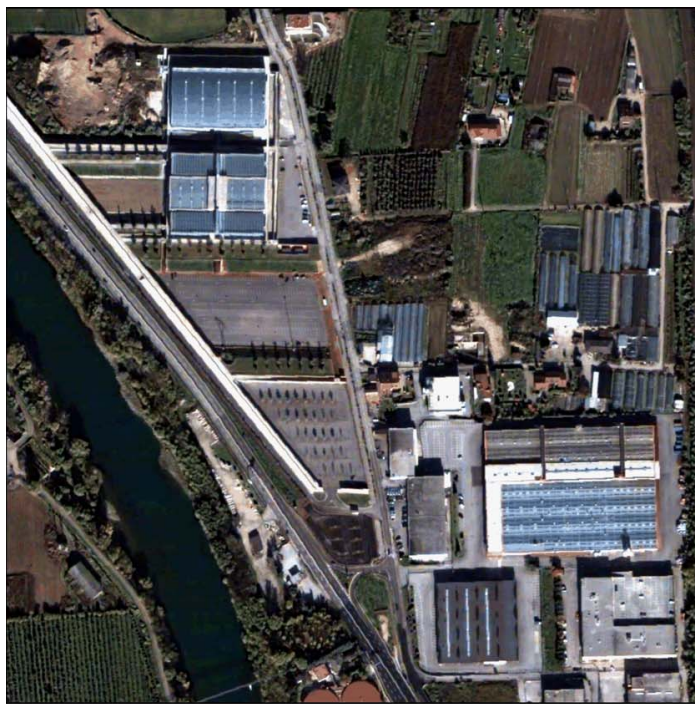

(a)

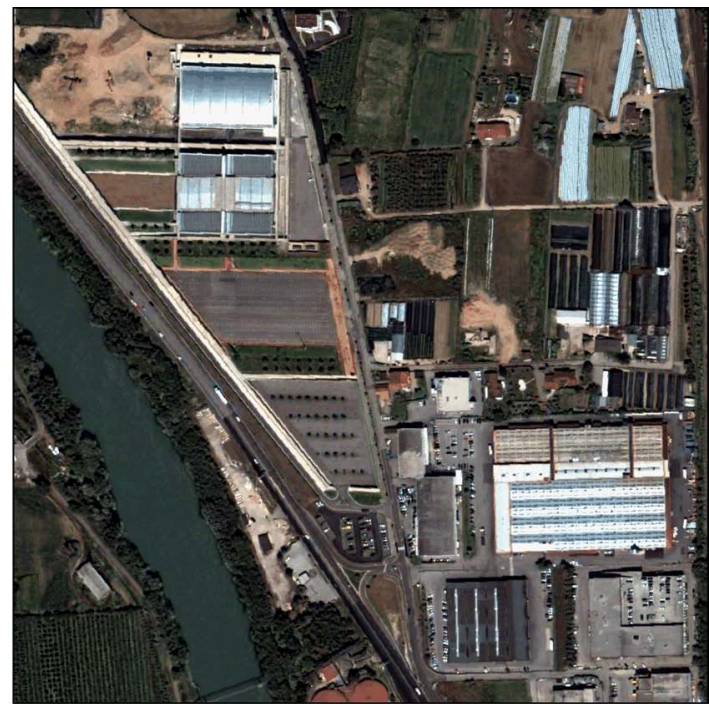

(b)

Fig. 14. Pansharpened images of the city of Trento (Italy) acquired by the Quickbird VHR multispectral sensor in (a) July 2005 and in (b) October 2006. Continuous white lines evidence changes in field crop, while the dashed lines mark changes in roofs (Test site 2).

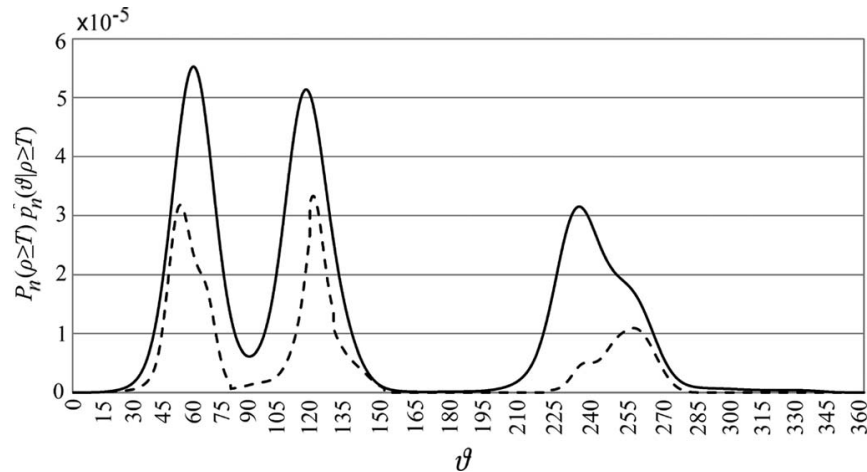

Fig. 15. Marginal weighted conditional densities $P_{\mathrm{n}}(\rho \geq T) \hat{p}_{\mathrm{n}}(\vartheta \mid \rho \geq T)$ of the direction in $A_{\mathrm{c}}$ (continuous line) at full resolution and (dashed line) at level four of the Daubechies SWT (Test site 2).

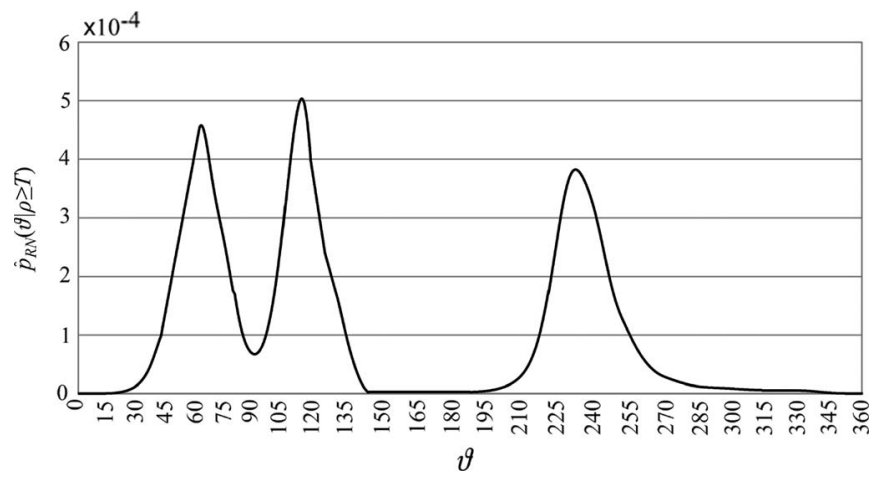

Fig. 16. Estimated conditional density $\hat{p}_{\mathrm{RN}}(\vartheta \mid \rho \geq T)$ of RN obtained with the proposed technique (Test site 2).

case, from an analysis of the behavior of this distribution, it is possible to identify three main modes, which represent the estimated sectors of dominant RN (this is confirmed from a visual analysis of polar scatterograms not reported for space constraints).

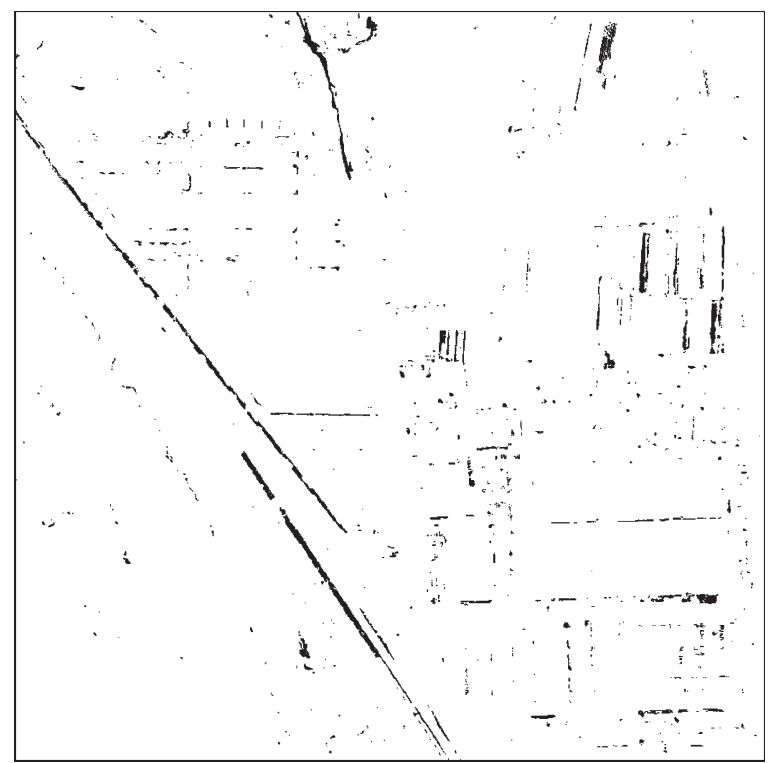

Fig. 17. RN map obtained by thresholding the $\hat{p}_{\mathrm{RN}}(\vartheta \mid \rho \geq T)$ obtained with the proposed technique (Test site 2).

We applied a threshold to the density of $\mathrm{RN}$ in order to estimate sectors of dominant RN. With a threshold equal to $1 \times 10^{-4}$ applied to $\hat{p}_{\mathrm{RN}}(\vartheta \mid \rho \geq T)$, and a threshold in the magnitude domain $T=310$, the sectors of dominant $\mathrm{RN}$ were identified between $35^{\circ}$ and $70^{\circ}$, between $90^{\circ}$ and $120^{\circ}$, and between $220^{\circ}$ and $250^{\circ}$. Fig. 17 shows the map of RN pattern obtained by thresholding $\hat{p}_{\mathrm{RN}}(\vartheta \mid \rho \geq T)$ with the proposed strategy. As for the previous test site, from a visual analysis of this map, it is possible to conclude that the regions identified as $\mathrm{RN}$ corresponds to areas of a misregistration, as they are mainly located in border of buildings, along the riverside and along the roads. In addition, also in this case, it is possible to note that the proposed technique allows one to properly distinguish between $\mathrm{RN}$ and true changes contributions in the estimation of $\hat{p}_{\mathrm{RN}}(\vartheta \mid \rho \geq T)$. 


\section{DISCUSSION AND CONCLUSION}

In this paper, we have analyzed the properties of RN in VHR remote-sensing images. This analysis was carried out in the context of a polar framework for CVA, where both the magnitude and the direction information of SCVs are represented. On the basis of the derived properties, a novel method for an adaptive estimation of the statistical distribution of $\mathrm{RN}$ in multitemporal VHR images has been proposed.

Images acquired by several sensors and with different landcover types were considered in the analysis (only a part of the results has been reported in this paper for space constraints). From them, some simulated data sets have been generated in order to study the effects of RN when: 1) the misregistration between the two considered images increases and 2) the resolution of the original images decreases. From this analysis, four different properties of the RN in VHR images have been derived, associated with both unchanged and changed pixels. These properties point out that misregistration may significantly affect the accuracy of change detection and show some important effects due to this specific kind of noise on VHR images. It is worth noting that on the basis of the conducted analysis, we can conclude that the properties of RN in VHR multispectral images are significantly different from those on high- or medium-resolution images. These differences should be properly understood and exploited in the mitigation of the effects of such a kind of noise in the definition of changedetection algorithms.

The analysis of the properties of RN resulted also in the definition of an adaptive technique for the estimation of the $\mathrm{RN}$ distribution in the polar domain. The proposed technique estimates the conditional density of RN with respect to the direction variable in the annulus of changed pixels, thus providing valuable information for the design of a change-detection procedure. In order to assess the reliability of the proposed estimation technique, we performed an analysis of the results obtained with the estimation method on a couple of test sites made up of two real multitemporal images acquired by the Quickbird sensor. These results confirm the effectiveness of the proposed technique in identifying and modeling $\mathrm{RN}$ also in presence of real multitemporal noisy images acquired under different conditions.

Even if the proposed technique exploits a multiscale decomposition for identifying $\mathrm{RN}$ and modeling its conditional distribution, the resulting estimate represents the behavior of the RN at full resolution. Thus, the estimated distribution can be used for analyzing the images at full scale, as the low-pass component used in the proposed strategy does not affect the scale of the estimation.

It is worth noting that depending on the considered scene, a slight relative shift effect (bias) on the direction variable between the distributions of the SCVs in the annulus of changed pixels at full and reduced resolution might be observed. This is due to the low-pass operation associated with the downscaling process. Even if this shift in general is not expected to be critical for the estimation technique, a simple correction procedure could be applied to the distributions before deriving the RN estimation.
The proposed strategy focuses on the estimation of the distribution of RN in the annulus of changed pixels and neglects the components of RN in the circle of unchanged pixels (whose properties are however identified in Section IV). Nonetheless, this is not critical because only the RN components in the annulus of changed pixels affect the change-detection map resulting in a significant false-alarm rate.

As a final remark, it is important to observe that the proposed strategy can be considered also for estimating the RN on medium- and high-resolution multispectral images. Indeed, even if the typical uniform distribution of $\mathrm{RN}$ in the annulus of changed pixels obtained with such kind of data [14] is against the assumption to have well-defined clusters of dominant RN in the annulus of changed pixels, the rationale inspiring the proposed estimation strategy is still valid.

As future developments of this paper, we plan to fully exploit both the derived properties and the technique for the estimation of the RN distribution to develop: 1) effective change-detection methods for VHR images based on both the Bayesian decision theory and context-sensitive strategies and 2) adaptive coregistration strategies based on the estimated local behavior of the RN.

\section{REFERENCES}

[1] L. Brown, "A survey of image registration techniques," ACM Comput. Surv., vol. 24, no. 4, pp. 325-376, Dec. 1992.

[2] B. Zitova and J. Flusser, "Image registration methods: A survey," Image Vis. Comput., vol. 21, no. 11, pp. 977-1000, Oct. 2003.

[3] L. Fonseca and B. Manjunath, "Registration techniques for multisensory remotely sensed imagery," Photogramm. Eng. Remote Sens., vol. 62, no. 9, pp. 1049-1056, 1996.

[4] A. Goshtasby and J. Le Mogne, "Special issue on image registration," Pattern Recognit., vol. 32, no. 1, pp. 1-150, 1999.

[5] G. J. Wen, J. J. Lv, and W. X. Liu, "A high-performance feature-matching method for image registration by combining spatial and similarity information," IEEE Trans. Geosci. Remote Sens., vol. 46, no. 4, pp. 12661277, Apr. 2008.

[6] X. Dai and S. Khorram, "The effects of image misregistration on the accuracy of remotely sensed change detection," IEEE Trans. Geosci. Remote Sens., vol. 36, no. 5, pp. 1566-1577, Sep. 1998.

[7] J. R. G. Townshend, C. O. Justice, and C. Gurney, "The impact of misregistration on change detection," IEEE Trans. Geosci. Remote Sens., vol. 30, no. 5, pp. 1054-1060, Sep. 1992.

[8] L. Bruzzone and R. Cossu, "An adaptive approach for reducing registration noise effects in unsupervised change detection," IEEE Trans. Geosci. Remote Sens., vol. 41, no. 11, pp. 2455-2465, Nov. 2003.

[9] H. Wank and E. C. Ellis, "Image misregistration error in change measurements," Photogramm. Eng. Remote Sens., vol. 71, no. 9, pp. 1037-1044, Sep. 2005.

[10] M. Beauchemin and K. B. Fung, "An adaptive filter for the reduction of artifacts caused by image misregistration," in Int. Workshop Anal. MultiTemporal Remote Sens. Images, May 16-18, 2005, pp. 174-176.

[11] D. A. Stow, "Reducing the effects of misregistration on pixel-level change detection," Int. J. Remote Sens., vol. 20, no. 12, pp. 2477-2483, Aug. 1999.

[12] L. De Carvalho, F. W. Acerbi, J. Scolforo, J. De Mello, and A. De Oliveira, "Wavechange: A procedure for change detection based on wavelet product spaces," in Int. Workshop Anal. Multi-Temporal Remote Sens. Images, Jul. 18-20, 2007, pp. 1-5.

[13] P. Gong, E. F. Ledrew, and J. R. Miller, "Registration-noise reduction in difference images for change detection," Int. J. Remote Sens., vol. 13, no. 4, pp. 773-779, Mar. 1992.

[14] F. Bovolo and L. Bruzzone, "A theoretical framework for unsupervised change detection based on change vector analysis in polar domain," IEEE Trans. Geosci. Remote Sens., vol. 45, no. 1, pp. 218-236, Jan. 2007.

[15] S. G. Mallat, "A theory for multiresolution signal decomposition: The wavelet representation," IEEE Trans. Pattern Anal. Mach. Intell., vol. 11, no. 7, pp. 674-693, Jul. 1989. 
[16] F. Bovolo and L. Bruzzone, "A detail preserving scale-driven approach to change detection in multitemporal SAR images," IEEE Trans. Geosci. Remote Sens., vol. 43, no. 12, pp. 2963-2972, Dec. 2005.

[17] A. W. Bowman and A. Azzalini, Applied Smoothing Techniques for Data Analysis: Kernel Approach With S-Plus Illustrations. Oxford, U.K.: Clarendon, 1997.

[18] E. Parzen, "On estimation of a probability density function and mode," Ann. Math. Stat., vol. 33, no. 3, pp. 1065-1076, Sep. 1962.

[19] E. A. Patrick and F. P. Fischer, III, "A generalized k-nearest neighbor rule," Inf. Control, vol. 16, no. 2, pp. 128-152, Apr. 1970.

[20] D. L. Reilly, L. N. Cooper, and C. Elbaum, "A neural model for category learning," Biol. Cybern., vol. 45, no. 1, pp. 35-41, Aug. 1982.

[21] L. Bruzzone and D. F. Prieto, "Automatic analysis of the difference image for unsupervised change detection," IEEE Trans. Geosci. Remote Sens., vol. 38, no. 3, pp. 1171-1182, May 2000.

[22] L. Bruzzone and D. F. Prieto, "An adaptive semiparametric and contextbased approach to unsupervised change detection in multitemporal remote-sensing images," IEEE Trans. Image Process., vol. 11, no. 4, pp. 452-466, Apr. 2002.

[23] F. Bovolo, L. Bruzzone, L. Capobianco, A. Garzelli, S. Marchesi, and F. Nencini, "Change detection from pansharpened images: A comparative analysis," ESA-EUSC: Image Inf. Mining, Mar. 4-6, 2008. [Online]. Available: http://earth.esa.int/rtd/Events/ESA-EUSC_2008/Papers/Ar28_ Garzelli.pdf

[24] L. Bruzzone and D. F. Prieto, "An adaptive parcel-based technique for unsupervised change detection," Int. J. Remote Sens., vol. 21, no. 4, pp. $817-822,2000$.

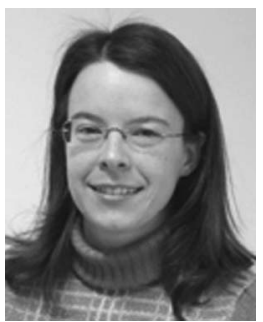

Francesca Bovolo (S'05-M'07) received the Laurea (B.S.) and the Laurea Specialistica (M.S.) degrees (summa cum laude) in telecommunication engineering and the Ph.D. degree in communication and information technologies from the University of Trento, Trento, Italy, in 2001, 2003, and 2006, respectively

She is currently a Postdoctoral member with the Remote Sensing Laboratory, Department of Information Engineering and Computer Science, University of Trento. Her main research activity is in the area of remote-sensing image processing. In particular, her interests are related to multitemporal remote-sensing image analysis and change detection in multispectral and SAR images, and very high resolution images. She conducts research on these topics within the frameworks of several national and international projects.

Dr. Bovolo is a referee for the IEEE TRAnsactions on GeOscience And Remote Sensing; IEEE Geoscience and Remote Sensing LETTERS; IEEE JOURNAL OF SELECTED TOPICS IN APPLIED EARTH ObSERVATIONS AND REMOTE SENSING; International Journal of Remote Sensing; Pattern Recognition; Remote Sensing of Environment; Photogrammetric Engineering and Remote Sensing; Photogrammetry and Remote Sensing; IEEE TRAnsactions on Aerospace and EleCtronic Systems; Sensors. She ranked first place in the Student Prize Paper Competition of the 2006 IEEE International Geoscience and Remote Sensing Symposium (Denver, August 2006). Since 2006, she has been with the Scientific Committee, SPIE International Conference on "Signal and Image Processing for Remote Sensing." She has served on the Scientific Committee of the IEEE Fourth and Fifth International Workshop on the Analysis of MultiTemporal Remote Sensing Images (MultiTemp 2007 and 2009).

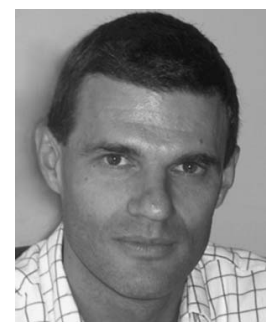

Lorenzo Bruzzone (S'95-M'98-SM'03) received the Laurea (M.S.) degree (summa cum laude) in electronic engineering and the Ph.D. degree in telecommunications from the University of Genoa, Genoa, Italy, in 1993 and 1998, respectively.

From 1998 to 2000, he was a Postdoctoral Researcher with the University of Genoa. Since 2000 he has been with the University of Trento, Trento, Italy, where he is currently a Full Professor of telecommunications. He teaches remote sensing, pattern recognition, radar, and electrical communications. He is the Head of the Remote Sensing Laboratory, Department of Information Engineering and Computer Science, University of Trento. He is an Evaluator of project proposals for many different governments (including the European Commission) and scientific organizations. He conducts and supervises research on these topics within the frameworks of several national and international projects. He is a Referee for many international journals and has served on the Scientific Committees of several international conferences. He is the author (or coauthor) of 74 scientific publications in referred international journals, more than 140 papers in conference proceedings, and seven book chapters. His current research interests are in the area of remote-sensing image processing and recognition (analysis of multitemporal data, feature extraction and selection, classification, regression and estimation, data fusion, and machine learning)

Dr. Bruzzone is a member of the Managing Committee of the Italian InterUniversity Consortium on Telecommunications and a member of the Scientific Committee of the India-Italy Center for Advanced Research. He is also a member of the International Association for Pattern Recognition and of the Italian Association for Remote Sensing. Since 2009, he has been a member of the Administrative Committee of the IEEE Geoscience and Remote Sensing Society. He ranked first place in the Student Prize Paper Competition of the 1998 IEEE International Geoscience and Remote Sensing Symposium (Seattle, July 1998). He was a recipient of the Recognition of IEEE TRANSACTIONS ON Geoscience AND Remote SENSING Best Reviewers in 1999 and was a Guest Editor of a Special Issue of the IEEE TRANSACTIONS ON GEOSCIENCE AND REMOTE SENSING on the subject of the analysis of multitemporal remotesensing images (November 2003). He was the General Chair and Cochair of the First and Second IEEE International Workshop on the Analysis of Multitemporal Remote-Sensing Images (MultiTemp), and is currently a member of the Permanent Steering Committee of this series of workshops. Since 2003, he has been the Chair of the SPIE Conference on Image and Signal Processing for Remote Sensing. From 2004 to 2006, he served as an Associated Editor of the IEEE GEOSCIENCE AND REMOTE SENSING LETTERS, and currently is an Associate Editor for the IEEE TRANSACTIONS ON GEOSCIENCE AND REMOTE SENSING.

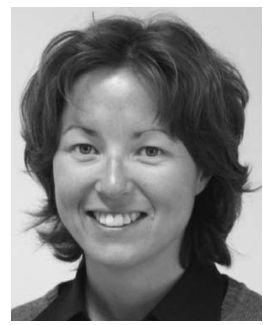

Silvia Marchesi (S'08) received the Laurea (B.S.) and Laurea specialistica (M.S.) degrees (summa cum laude) in telecommunication engineering from the University of Trento, Trento, Italy, in 2005 and 2007, respectively, where she is currently working toward the $\mathrm{Ph} . \mathrm{D}$. degree in information and communication technologies.

She is currently with the Remote Sensing Group, Department of Information Engineering and Computer Science, University of Trento. Her main research activity is in the area of remote-sensing image processing; in particular, her interests are related to change detection in very high geometrical resolution images. 